\title{
Modeling representational shifts in learning the number line
}

\author{
Leif K. Lende (leif.k.lende@ diakonhjemmet.no) \\ Department of Social Education and Occupational Therapy, \\ Diakonhjemmet University College Rogaland \\ Vaagsgaten 40, N-4306 Sandnes, Norway
}

\author{
Niels Taatgen (…a.taatgen@ rug.nl) \\ Department of Artificial Intelligence, \\ University of Groningen \\ Nijenborgh 9, 9747 AG Groningen, Netherlands
}

\begin{abstract}
On the basis of findings from an experiment with 6-year-old children we show a proposal for a cognitive model of representational shifts in learning the number line. The findings from the experiment provide information on number line estimation - that is, translating a number to a spatial position on a number line. Though the experiment is a replication of an experiment done by Siegler and Ramani (2008) where they concluded with a logarithmic to linear shift, we could not find logarithmic representation of the results from any of our subjects. What we find is anchor points as important for improvement on learning the number line.
\end{abstract}

Keywords: Learning; numerical magnitudes; number line; dynamic decision making; memory; cognitive architectures; ACT-R.

\section{Introduction}

In this paper we present a model of the learning process involved when dealing with the estimation of what position a number value has on a number line.

The learning sequence involved is the one that Siegler calls the logarithmic-to-linear shift in representations of numerical magnitude (Siegler, Thompson, \& Opfer, 2009).

Siegler et al (2009) show that children undergo parallel changes from logarithmic to linear representation on numerosity estimation tasks.
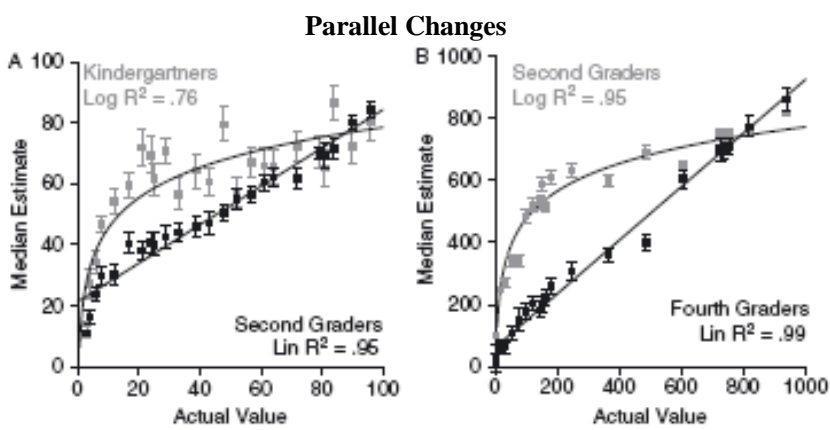

Figure 1. The logarithmic to linear shift. From Siegler, Thompson, \& Opfer, (2009), Copyright 2009 Wiley. Reprinted with permission.

The example we have reused from their article in figure 1 shows long-term changes in estimation of whole number magnitudes. (A) On 0-100 number lines, kindergartners' estimates were better fit by the logarithmic function than by the linear, whereas second-graders' estimates were better fit by the linear function than by the logarithmic; (B)
On 0-1000 number lines, second-graders' estimates were better fit by the logarithmic function than by the linear, whereas fourth-graders' estimates were better fit by the linear function than by the logarithmic.

The explanation by Siegler et al. was challenged by others (Barth \& Paladino, 2011). They point out that one of the challenges of putting a number on the number line is to have a sense of proportion: what exactly is the length of a single unit? This is not a trivial question for children that do not yet have a sense of what division is.

Our own earlier work also showed that a simple Weber explanation of the learning sequence of the logarithmic to linear shift does not hold as a complete explanation (Lende \& Taatgen, 2011). We proposed that a possible account for the transition towards a linear representation is that children learn the location of particular points on the number line. Schneider et al. (2008) showed that the distribution of fixations on the number line for all three groups of first grade, second grade and third grade children are concentrated around beginning, midpoint and ending of the number line, suggesting that at least these three points are represented separately (Figure 2).

\section{Distribution of fixations}

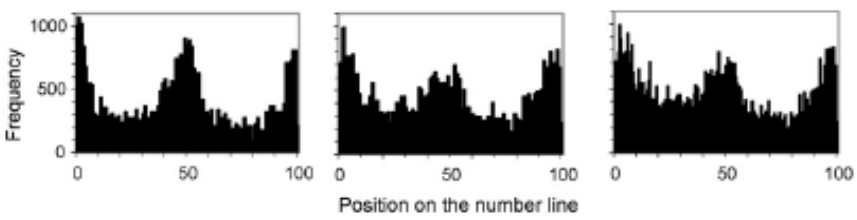

Figure 2: Distribution of fixations on the number line (left: first grade; middle: second grade; right: third grade). From Schneider et al. (2008), Copyright 2008 Elsevier.

Reprinted with permission.

In addition, their work shows that from grade 1 to 3 children tend to increasingly focus on the correct positions on the number line while solving the estimation tasks. .

Because of the mentioned challenges to the explanation of Siegler et al. and that it is hard to see from aggregated data what is going on with individuals; we have designed our experiment as a replication of Siegler and Ramani (2008) with the goal to look at individuals and the goal to build a model.

\section{The number line estimation task}

The experiment is a replication of a study by Siegler and Ramani (2008) among preschool children from low 
income families. Siegler and Ramani found a striking improvement in number-line performance in the children after they had played a board game involving counting, but not on a board game involving colors.

\section{The Outline of the experiment}

The experiment consisted of four elements: a pretest, a training program of two weeks, a posttest and finally a second post test to measure long-term learning. We will not discuss the results of the second post test here.

After the pretest, the sample group was provided with the same training program as Ramani and Siegler used for their test of preschoolers (Siegler \& Ramani, 2008). Children met one-on-one with an experimenter for four 15minute sessions within a 2 -week period. After the 2 weeks the first posttest was conducted. Then after seven new weeks a second posttest was conducted. All tests are the same.

\section{The Method of the three tests \\ Participants}

Participants were 39 Norwegian children in their first year of school, so-called preschool, with no experience with number lines. All of them are born in 2004 and recruited from the same municipality, Gjesdal. 17 of them are recruited from Solås School, 7 from Dirdal School and 15 from Bærland School. The population at these schools is mixed, but at Bærland with a larger representation of bilingual children, Norwegian not being their mother tongue. 21 of the participants participated in the experiment while the rest of them acted as a control group.

\section{Materials}

Stimuli for the number line estimation task were two stacks of 10 sheets of paper, each with a $25 \mathrm{~cm}$ long line arranged horizontally across the page, with ' 0 ' just below the left end of the line, and ' 10 ' just below the right end. A number from 1 to 10 inclusive was printed approximately 3 $\mathrm{cm}$ above the center of the line, with each number printed on one of the 10 sheets in each stack. The order of the sheets in the stack was randomized.

\section{Procedure}

The test is conducted as a teacher to student task:

- The teacher or student pulls a sheet from the stack.

- The teacher says: "Here is the number [number that is on the pulled sheet]. And here you see a line that starts with 0 and ends at 10 . Where on this line is the correct position for the number you see? Put a mark with your pencil".

- The student makes a mark where he or she thinks the number should be positioned. There is no time constrain for the subject to fulfill the task.

The task is carried out with all the sheets in the first stack. Then the task is continued in the same way with the second stack. In this way the numbers from 1 to 10 inclusive were presented twice in random order, with all numbers presented once before any number was presented twice. No feedback was given, only general praise and encouragement.

\section{Method of the Board Game}

In the training program between the pretest and the first posttest the subjects played a board game using a play button to move along a line of squares from square to square.

\section{Materials}

The board game for the experiment group shown in figure 3 consists of a number line with numbers in colored squares from 1 to 10 with a blank square as starting position for the game.

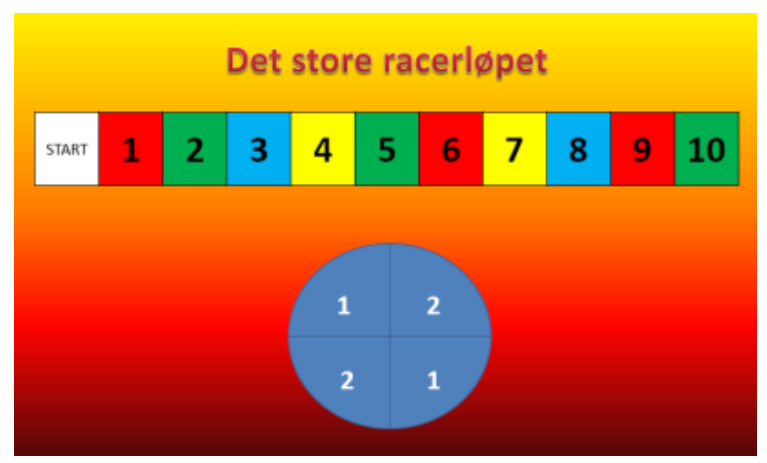

Figure 3. The Game board for the experiment group

Beneath the number line there is a circle with a spinner. (The spinner is not shown on the figure) In each quarter of the circle the numbers one or two is printed.

The board game for the control group consists of a similar line of squares, but with no numbers as shown in figure 4.

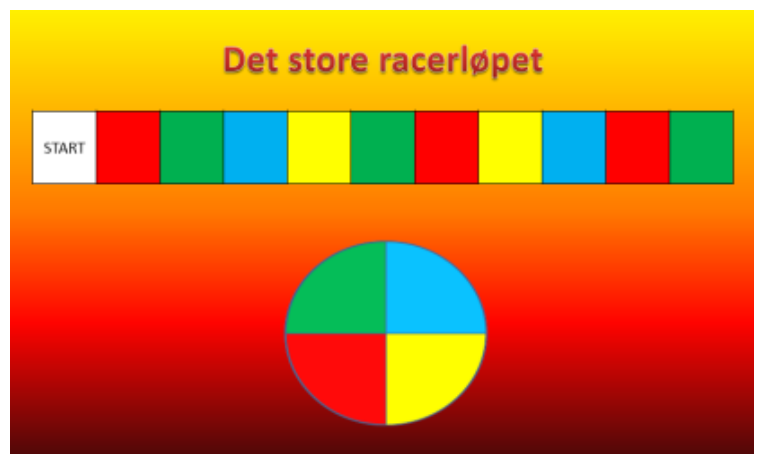

Figure 4. The Game board for the control group

Beneath the line of colored squares there is a circle with a spinner. (The spinner is not shown on the figure) Each quarter of the circle is painted with different colors corresponding to the colors used in the line of squares.

\section{Procedure}

The subjects trained with their board games for 15 minutes twice a week for 2 weeks. 
When a subject of the experiment group turns the spinner the player moves his play button as many squares as the spinner tells ( 1 or 2 steps) while saying out loud the numbers in the squares he steps on.

When a subject of the control group turns the spinner the player moves his play button to the first square on the line of squares that is painted with the same color as given by the spinner.

\section{Result and discussion}

Figure 5 and 6 show the mapping between numbers and positions on the number line that we found in the pretest and the first posttest of the experiment. Performance is on average reasonably good.

It is surprising that where the curve differs from linear, it is not towards a logarithmic curve, but in the opposite direction..

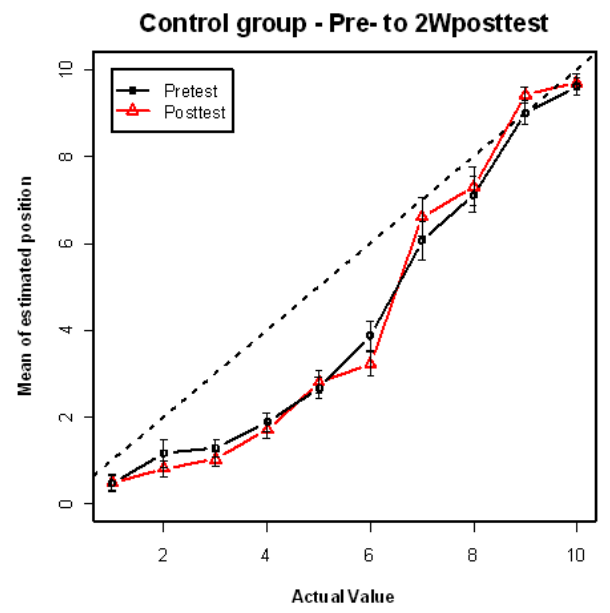

Figure 5. The figure shows the average result of the control group positioning the numbers on the number line. Points are plotted with error bars.

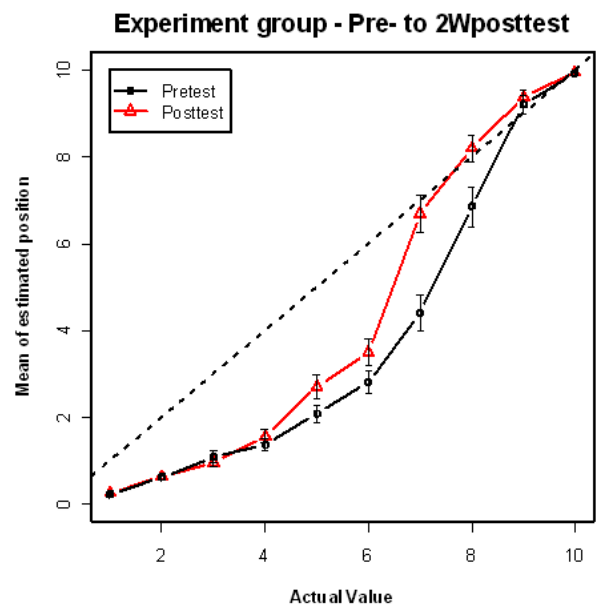

Figure 6. The figure shows the average result of the experiment group positioning the numbers on the number line. Points are plotted with error bars.
The fact that the results are neither linear nor logarithmic is surprising. Inspections of individual subjects (see Figure 8 later in the paper) show that individual estimates have strong linear trends, only not with the right slopes. This suggests that subjects use some sort of counting strategy, but with a counting unit that is not a tenth of the whole line, but rather a smaller unit.

Figures 5 and 6 suggest that the experimental manipulation was indeed successful. To analyze this we performed a two-way Anova with the summed error as the dependent variable and condition and pre- vs. posttest as independent variables. This produces an interaction effect between condition and test, $F(1,1441)=6.02, p=0.014$, and a main effect of test, $F(1,1441)=7.84, p=0.005$, but no main effect of condition, $F<1$. This means that the experimental group does indeed improve more on the posttest than the control group. Figure 6 shows that this improvement is mainly on the numbers 5 through 8 .

To have a better picture of individual differences in the learning process, we used the k-means clustering algorithm (MacQueen, 1967) with as input the difference between the pre- and post-test of the accuracies of each of the ten numbers. The result of the cluster analysis of this combined group of both experiment group and control group indicates that there are two different patterns of improvement: one for numbers around five and six, and one for the numbers around eight (Figure 7).

Individuals in the first cluster (red circles) include six subjects from the experiment group and only two from the control group. This indicates that several more individuals in the experiment group have made improvement on the numbers 5-7 than those in the control group. The second cluster (green triangles) corresponds to no or little improvement, and includes 13 subjects from the control group and 8 subjects from the experiment group. And in the third cluster (black plus signs) there are three subjects from the control group and five from the experiment group.

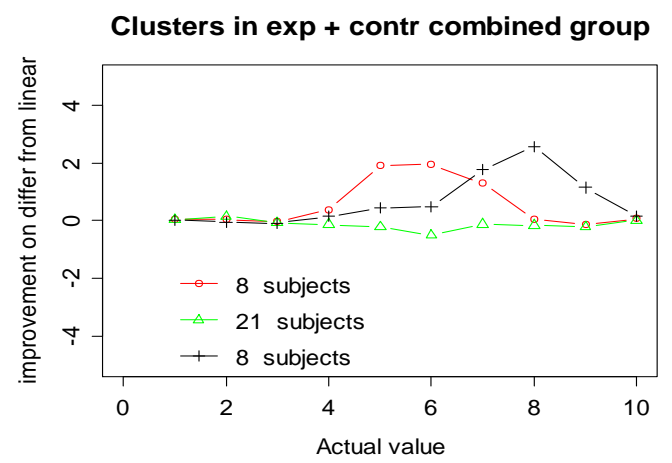

Figure 7. The graph shows the result of the cluster analysis on the improvement of distance from a true linear representation between pretest and posttest. Positive values indicate improvement and negative values the opposite. 
To conclude, the data tell us a number of things. First, some sort of counting seems to be used to arrive at a point, but not with the correct counting unit. Second, improvements in performance seem to be centered around the middle point of the number line and towards the end of the number line, but hardly at the beginning. This suggests that subjects do not improve the length of their counting unit, but rather in the way they use it. Improvements around the middle of the number line suggest they learn that five is in the middle of the line and can be used as a starting point for counting. Improvements towards the end of the line suggests subjects learn that the higher numbers, 7 and 8 in particular, are close to 10 , so that counting back from 10 is a better strategy than counting up from zero.

\section{The model}

A possible model of progressing towards a linear time scale can therefore be one that increasingly learns the locations of particular points on the number line, and uses those as anchors to determine the points that it does not know. It therefore needs some sort of representation of the positions of anchor points, but also a method for determining points in between those anchors by counting.

As a theory of how anchor points are stored in memory, we use ACT-R's declarative memory (Anderson, 2007). In order to determine positions between the anchor points, we use two mechanisms. The first one is a retrieve function that decides which anchor point will be the starting point. The second one is a count mechanism that uses a count unit to count up or down from the starting point to decide the position for the number on the number line. The initial size of the count unit is decided by average size from real data and randomly varies in size according to variation found between subjects in real data.

\section{The details of the model}

The basic assumption of the model is that the subjects already know how to count from 1 to 10 , but that they have incomplete knowledge of how to put those numbers on the number line. An anchor point represents knowledge about putting numbers on the line and is expressed by associations between a number and a position on the line. In most cases this knowledge only consists of the number zero and the number ten on the extreme ends of the line, but may also consist of the middle point five.

To represent the different levels of knowledge about numbers and anchor points, we vary the base-level activation of the chunks associated with them.

If the model has to put a particular number on the number line, it tries to retrieve an anchor point from declarative memory for the number. If there is no direct match between any of the available anchors, to process of partial matching will retrieve the anchor point that has the highest activation. This activation depends on two aspects: the base-level activation of that anchor, and its similarity to the request number. So if the model tries to retrieve the number 6 and only 0 and 10 are available as anchors, the model might retrieve 10 because it is closer to 6 , but also 0 because that point has a higher base-level activation.

Whenever the model retrieves an anchor that is not already the number that it is trying to retrieve, it will apply counting to reach the desired point on the line. However, the unit of counting, following our data, is smaller than an actual tenth of the length of the line $(0.42 \mathrm{~cm} \pm 20 \%)$.

By simply varying the base-level activations of the anchors, we can reproduce most of the patterns of responses that we see in the data.

The model has an activation baseline function and there are three functions dealing with the declarative memory.

One function makes a reference list of numbers involved and their position on the number line. A chunk is represented as a list, with a number (what number is it about) and a position (where is it on the number line), and a reference list with moments in time the chunk has been accessed.

The mismatch function is based on Weber's law, and the result value is zero, a negative value or a positive value depending on whether the first number is similar, smaller than or larger than the second number. The mismatch assumes two numbers are more similar if they are closer and higher and is used to calculate the activation of a chunk.

A retrieval function is performing the retrieval and adds noise. Because of that we do not use the regular ACT-R retrieval rule and noise activation function.

Another function takes care of the counting procedure. The counting unit has an initial length correlating to the mean of the length of count units found in the real life data set from our experiment. The length of this unit is randomly shorter or longer for each individual simulation according to variation found in real life data. The same is done with the count unit for each count step.

In this same function simple proportioning is implemented the way that proportioning is activated after simulation of an individual's trial number 150 .

\section{Results from running the model and discussion}

When we run the model simulating a subject doing a certain number of trials, basic-level activation is not increased after every trial. The trials represent the training with the board game in real life. We assume that only after dozens of times of playing this game a subject obtains the kind of new crucial knowledge that makes a shift in numerical representation on the number line. This new knowledge could be that the position of a certain number is either at the beginning or at the end of the number line. In our experiment those numbers are 1 (or 0 ) and 10 , the start point and the endpoint. So for the number at the endpoint there is no need for counting upwards from 0 anymore. We have got what we call a representational shift and the number at the endpoint has got a stronger activation as anchor point. That is why we let the model also run dozens of trials before each increase of base-level activation. The increase of base-level activation is done by 
adding entries in the chunk for the appropriate anchor point.

Another example of such a representational shift is when a subject realizes that one or several numbers are close to 10 and counting downwards from 10 is how to position those numbers on the number line.

To what extent those shifts in knowledge represent different levels of knowledge is not clear, but we have made the assumption from our rather limited amount of real data that it could be that the first shift for children that have already learned to count, is to learn that the endpoint of the line is useful as an anchor point. In our experiment that is 10 . Next is that some numbers are close to ten, then that five is an anchor point, and last, that the counting unit has to be adapted to a reasonable size. Plotted images show the relation and progress between these shifts. And we can easily find related and rather similar images to each of those steps from values of individuals from empirical data, when plotted (See figure 8).
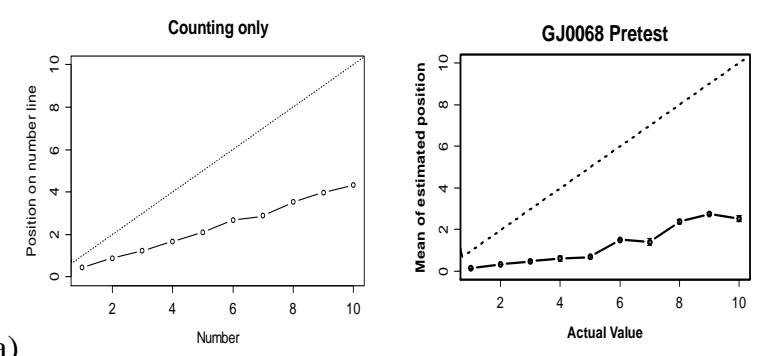

8 a)
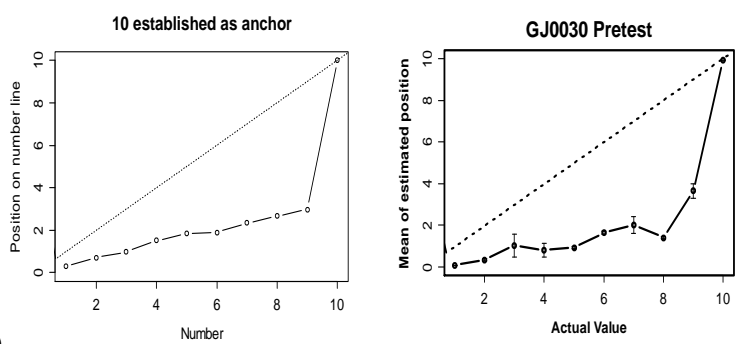

$8 \mathrm{~b})$
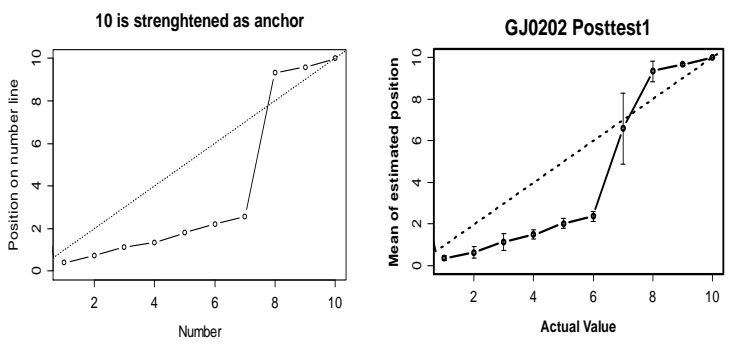

$8 \mathrm{c})$
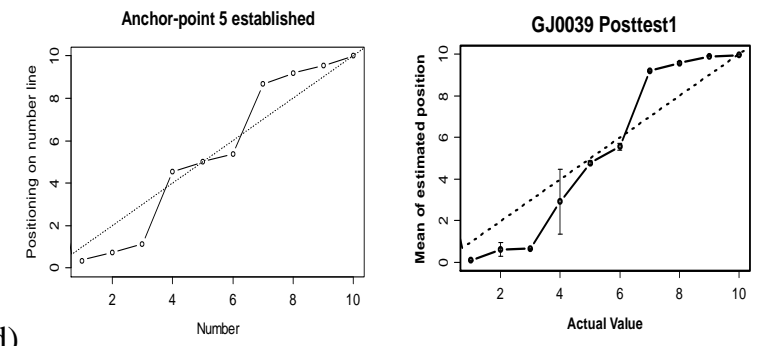
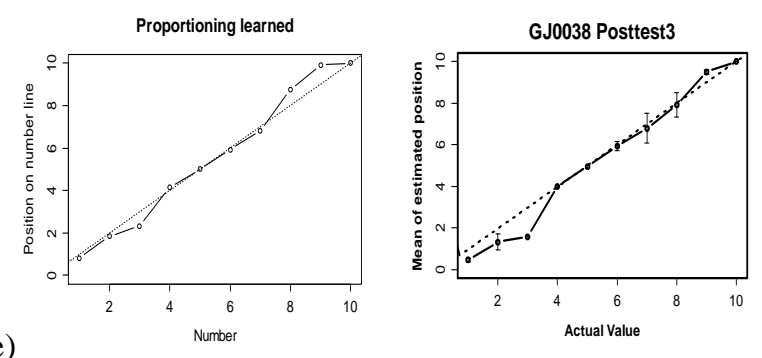

Figure 8 Model result from typical levels shown by model to the left and corresponding example from real data to the right: a): 1(or 0) is the only anchor point. b): Now knowing 10 as anchor point, c): Now knowing 10 even better, d): also knowing 5 as anchor point. e): Proportioning is activated.

When it comes to at what point a shift in knowledge should occur, in the model we have defined an amount of trials that we from our real data think is reasonably close to what we could find in real life.

A prior level of knowing how to represent numbers on an empty number line is of course when a child does not know how to do it at all. Siegler and Ramani (2008) show that even those at this prior level learned to deal with the number line during training with the board game. But for our model we have defined as the initial level when children know where 1 (or 0 ) is at the number line, and use counting only as strategy for putting other numbers on the right position.

The initial level, shown in figure 8 a), is a level where only counting is involved and base-level activation only on the chunk for the number 1 as an anchor point with a value of 1,15 . In this case the chunk for 10 only has a base-level activation of $-0,458$.

At the next stage, shown in figure $8 \mathrm{~b}$ ), which is after 60 trials, the base-level activation for the chunk of the number 1 is unchanged but for 10 it is increased to 0,640 The model now simulates where the anchor point 10 is, just like the subject GJ0030 at Pretest now knows where it is. .

After 100 trials we assume that a new shift occurs, shown in figure $8 \mathrm{c}$ ), Now the base level-activation for 1 is increased to 1,333 and for 10 to 0,928 . Just like the subject GJ0202 in Posttest1 now knows, the model now simulates that 8 and 9 is close to 10 and positions those numbers by counting down from 10 as anchor point. The next shift will occur in the model after 150 trials, shown in figure $8 \mathrm{~d}$ ). Base-level activation for 1 is unchanged, for 10 it is increased to 1,151 . The number 5 now, as a new anchor point, has a base-level activation of 1,151. And the model now simulates knowing the midpoint, which is five, as anchor point. In real life data we find a close case in subject GJ0039 at Posttest1.

The last shift implemented in our model so far, shown in figure $8 \mathrm{e}$ ), is when a subject obtains knowledge about the need for, and how to, adapt the counting unit to the most suitable size, In this case the base level activation is 
unchanged for all three anchor points, but proportioning of the counting unit is activated with some random errors.

The proportioning function of the model is rather preliminary and simply divides the physical length of the number line with the amount of numbers on line, which is 10 for this actual experiment, and adjusts it for error by randomizing according to what we find in real life data. Young children, as those in our experiment, do normally not obtain this level, and our experiment does not give us data for this. But we assume that what happens in real life is that finding a close to perfect size of the counting unit, is obtained either during training by trial and error or by dividing the line length in halves or thirds, one or several times.

In our data it seems that all of the subjects who understand the task use counting as an important part of the strategies for estimation. As we can see, in the same way as the results of our collected data from 6 year old children showed, we obtain no logarithmic curve from running our model. If we investigate the physical size of the unit used by the subjects in counting up or down from an anchor point, it is for all of them much smaller than a tenth of $25 \mathrm{~cm}$, which was the length of the number line used in the estimation task. But on the opposite, with a larger scale, for example up to 100, the child's unit will be too large, and counting will often lead to a logarithmic curve like Siegler and others has found.

This shows that for the counting strategy, most of the subjects do not have a clear clue of what the size of a unit should be.

A last comment to our model, is that obviously there are moments between those representational shifts that we have built our model on so far, where subjects in real life obtain brick stones of knowledge that prepare for the shifts. For example we assume that when playing the board game and moving from number to number, the subjects learn connections between numbers. And the activation of those connections may be strengthened almost every time they play the game. This issue is in focus for further development of the model.

\section{Conclusion}

From our findings in real data we have concluded that a logarithmic scale for a representation of the result of a number line task depends on the proportion between the counting units the individual uses and the length of the empty number line. In our experiment the unit is too small to lead to a logarithmic representation.

We found that what they actually learn from training with the board game, are that higher numbers are close to 10 and that 5 and 6 are approximately in the middle of the line.

It does not make sense to show average data from the real life data set, because the individuals are so different. However, we can find shifts in learning levels in different individuals.

Those different shifts are easily simulated by our model.

\section{Acknowledgments}

Thanks to Gjesdal municipality for permission to conduct, and funding of our experiment, provided by paying teachers (for putting in extra hours) to assist in our experiment.

\section{References}

Anderson, J. R. (2007). How can the human mind occur in the physical universe? (p. 290). Oxford University Press. Retrieved February 1, 2011

Barth, H. C., \& Paladino, A. M. (2011). The development of numerical estimation: evidence against a representational shift. Developmental science, 14(1), 125-35

Lende, L. K., \& Taatgen, N. A. (2011). Modeling the Logarithmic-To-Linear Shift In Representations Of Numerical Magnitudes. In L. Carlson, C. Hoelscher \& T. F. Shipley (Eds.), Proceedings of the 33rd Annual Conference of the Cognitive Science Society (pp. 207212). Austin: TX: Cognitive Science Society.

MacQueen, J. (1967). Some methods for classification and analysis of multivariate observations. In L. M. Le Cam \& J. Neyman (Eds.), Proceedings of the Fifth Berkeley Symposium on Mathematical Statistics and Probability (pp. 281-297). Berkeley, CA: University of California Press.

Schneider, M., Heine, a, Thaler, V., Torbeyns, J., Desmedt, B., Verschaffel, L., et al. (2008). A validation of eye movements as a measure of elementary school children's developing number sense. Cognitive Development, 23(3), 409-422.

Siegler, R. S., \& Ramani, G. B. (2008). Playing linear numerical board games promotes low-income children's numerical development. Developmental science, 11(5), 655-61.

Siegler, R. S., Thompson, C. a, \& Opfer, J. E. (2009). The Logarithmic-To-Linear Shift: One Learning Sequence, Many Tasks, Many Time Scales. Mind, Brain, and Education, 3(3), 143-150. 